\title{
Continuing Professional Development: Perspectives of Kenyan Nurses and Midwives
}

\author{
Janet Kemei, Josephine Etowa \\ School of Nursing, Faculty of Health Sciences, University of Ottawa, Ottawa, Canada \\ Email: jkemei233510@gmail.com
}

How to cite this paper: Kemei, J. and Etowa, J. (2021) Continuing Professional Development: Perspectives of Kenyan Nurses and Midwives. Open Journal of Nursing, 11, 121-131.

https://doi.org/10.4236/ojn.2021.113011

Received: January 26, 2021

Accepted: March 12, 2021

Published: March 15, 2021

Copyright (อ 2021 by author(s) and Scientific Research Publishing Inc. This work is licensed under the Creative Commons Attribution International License (CC BY 4.0).

http://creativecommons.org/licenses/by/4.0/

\section{(c) (i) Open Access}

\begin{abstract}
Background: The purpose of this paper is to report the perceptions of continuous professional development by the nurses and midwives in a low resource country. These findings are part of a larger study on the experiences of nurses and midwives providing postpartum care in rural areas of Kenya. Besides being the main healthcare providers in rural areas, nurses in low resource countries have limited access to continuous professional development. Research design: This is a qualitative research guided by critical theory and Foucault's concepts of power and knowledge. Focused ethnography provided the framework of data collection and analysis. 23 in-depth interviews were contacted, and thematic analysis was used for data analysis. Reflexive memos kept throughout the research process helped to ensure the trustworthiness of data. Findings: This paper will focus on the theme of continuing professional development (CPD) as a resource for a healthy work environment. While the knowledge of the nurses and midwives allowed them to provide care to the mothers and their infants adequately, personal and structural barriers prevented them from participating in continuing professional development. Discussion and Conclusion: A well-prepared nursing and midwifery workforce could have the capacity to transform the health outcomes of their clients through the application of evidence-based practices. Therefore, courses that are context-appropriate and meet the needs of different learners should be available to support the nurses' and midwives' professional growth. There is a need for more research on the nurses' and midwives' participation in CPD in Kenya, and the effect of CPD on specific health outcomes.
\end{abstract}

\section{Keywords}

Nursing, Midwifery, Continuing Professional Development, Low-Middle Income Countries 


\section{Background}

Nurses and midwives account for about $50 \%$ of the healthcare workforce globally, and they often serve as primary caregivers in rural health facilities [1]. They play a significant role in the contribution of the sustainable Development Goals (SDGs), including preventing maternal and infant morbidities and mortalities and improving mothers' and their infants' health outcomes [2] [3]. Therefore, achieving health for all will depend on well-trained, educated, regulated, and supported nurses and midwives [1]. Most nurses undergo three to four years of basic nursing training that prepares them for a lifetime career. Hence, continuous knowledge update through further professional education is necessary to ensure their continued competency and improve nursing knowledge essential for the health system's functioning [4].

With the ever-changing technologies and emergence of new knowledge, nurses and midwives must engage in continuing professional development (CPD) to keep their clinical skills updated and promote safe and effective care [5]. CPD learning is not always possible for the nurses and midwives in rural areas of low resource countries. "[6]" stipulates that the nurse carries personal responsibility and accountability for nursing practice, and for maintaining competence by continual learning. Therefore, $\mathrm{CPD}$ is an integral part of the nursing role in many nursing governing bodies. It facilitates team competence and promotes dialogue about the quality of knowledge and collaboration among the frontline healthcare providers [5].

Countries have different requirements for CPD. In Kenya, nurses and midwives complete at least 20 hours of (CPD) every year to maintain their registration every three years [7]. In the United Kingdom, the Nursing and Midwifery Board requires nurses and midwives to complete 35 hours of CPD every year. At least $20 \mathrm{hrs}$ must have included participatory learning, for example attending a conference or participating in a workshop [8]. The College of Nurses of Ontario (CNO) has not set minimum hours. It stipulates that nurses are accountable for determining their own learning needs and maintaining and continually improving their competence by participating in the College of Nurses of Ontario's Quality Assurance (QA) Program. The QA program promotes reflection by nurses' and peers' feedback to identify their learning relevant to their practice. It includes performing a self-assessment, seeking peer input, developing a learning plan, implementing and evaluating the procedure's outcomes [9].

Besides the CPD requirement in Kenya, there is no clear evidence that the nurses and midwives have an enabling environment to accomplish these requirements.

This paper aims to report part of the findings of the study; experiences of nurses and midwives providing postpartum care in rural Kenya. One of the six themes in this study, Fostering a Healthy Work Environment, illuminated the continuous development of knowledge and skills as a facilitator in creating a positive work environment for the nurses and midwives. Regardless, several individual and structural factors prevented nurses from participating in CPD. 


\section{Research Designs}

\subsection{Theoretical Perspectives}

The critical theory [10] and Foucault's concepts of power and Knowledge [11] guided this study. These theories illuminated how the complex contextual factors intersected to determine nurses and midwives' reality in rural Kenya. CT stance is that a comprehensive reality has been shaped by socio-economic, political, and cultural factors that are now considered real, with the facts constructed by those in power at history points [10]. Research that seeks to unearth the inequities and call for action could change these realities based on power and identity struggles [12]. Foucault understands power as a non-entity that produces reality with individuals being vessels of knowledge production [11]. Foucault's concepts of power and knowledge were relevant to this inquiry. Nurses and midwives could be in a position of power under their professional experience and underprivileged based on the history of the nursing profession's development [13].

\subsection{Methodology}

Focused ethnography (FE) informed the study design. FE is a more targeted form of ethnography where inquiry is conducted in a natural setting and seeks to acquire knowledge by focusing on particular elements in society, to address specific problems [14]. Unlike traditional ethnography that explores knowledge from the entire society, FE centers on the specific topic among a distinct group of persons, hence it is used to conduct research that aims to inform decision-making regarding a particular problem. For example, in this study, FE allowed a close examination of how several factors influenced nurses' and midwives' day-to-day work and potentially affected the health outcomes of mothers and infants in rural Kenya. The study emphasized the experiences of nurses and midwives as individual entities as well as located them within the health care professional group. With $\mathrm{FE}$, the researchers can illuminate cultural phenomenon and health practices, and how they affect diverse cultures [14]. "[15]" explains that FE involves a limited number of participants, and research is problem-focused and context-specific, time-limited, with participants holding specific knowledge, and does not require prolonged observation in the field.

The researcher obtained ethics approval from the University of Ottawa Research Ethics Board (REB) in June 2017 and the Institutional Research and Ethics Committee (IREC) in Kenya in May 2017. The Medical Superintendent at the Kapsabet County Referral Hospital and the Nandi County Director of Health provided letters approving research.

With permission from the health facilities' authority, the researcher posted recruitment posters at the health facilities. The study used Purposeful sampling [16] to recruit participants, and snowballing occurred when participants referred their colleagues who met the inclusion criteria. Twenty-three nurses and midwives who worked in nine health centres and the referral hospital participated in the study. With their consent, the researcher held an in-depth individual inter- 
view session with each participant at a private venue of their choice. The interview guide contained semi-structured open-ended questions that were developed using information gathered during the literature review [17]. The questions on the interview guide addressed the overall study aim and objectives. It covered several topics including the nurses and midwives' perspectives on postpartum care, available postpartum services in Kenyan rural communities, resources available for postpartum service provision, health care policies influencing postpartum, their career goals and training needs, as well as suggestions for improving postpartum care. The researcher guided conversations and probed participants for incomplete sentences. Each participant received an honorary fee at the beginning of the interview session.

Data collection and analysis occurred concurrently [18]. CT and Foucault's concept of power and knowledge, together with the investigator's experiences working in Kenya and North America, helped shape the meaning of the words during data analysis. Credibility, transferability, dependability, and confirmability were the aspects of trustworthiness used throughout the research process [19]. The investigator also engaged in reflexibility [20] as a conscious acknowledgement of the research process. Reflexivity was critical to avoid misrepresentation of the participants [21].

\section{Findings}

The sub-theme of "Continuing professional knowledge and skills development" elucidates how nurses and midwives obtained current knowledge for clinical practices such as identifying, assessing, and treating complications during the postpartum period. The participants in this study used the term continuing medical education (CME) to describe the on-the-job training that the nurses and midwives and other healthcare professionals received periodically to enhance their knowledge in the current competencies required to deliver quality, good healthcare services.

The health care professionals undertook education in different ways such as one-time classroom education, over some time course training, post-incident huddles or debriefings, pamphlets, or as life exercises or drills. Participants emphasized the importance of the nurses and midwives to meet these postpartum emergency demands regardless of knowledge delivery. Continuing professional education created a healthy work environment for the nurses and midwives, and the knowledge allowed them to provide care to the mothers and their infants adequately.

Most of the nurses and midwives working with mothers have received some basic training on how to deal with obstetric emergencies by completing courses offered by non-governmental organizations, but a few nurses and midwives have been trained in dealing with complicated obstetric emergencies. (P002)

Some facilities had an education committee that conducted training through huddles following the management of any rare complication since this prepared 
the nurses and midwives for similar situations.

There is a committee that coordinates the CME topics ... we suggest to this committee the subjects that we wish discussed and they arrange a time for training ... Sometimes, working in a health center without a doctor, there are difficult cases. When we have a difficult and rare case like cord prolapse, we discuss so that we gain more knowledge about it ... it is better to know how to deal with a case so that when you are alone, you know what to do. (P020)

Having the expertise of dealing with obstetric complications is particularly important when working in rural areas since most mothers, especially those who delivered at home would typically seek skilled healthcare only after complications to either the mother or the newborn. Nurses and midwives were empowered and confident when treating these disorders after they had received training on emergency obstetric complications.

Most of the referrals have life-threatening complications like postpartum hemorrhage. Some of us have been given some training in dealing with these cases. Therefore, when we receive them, we take the initiative to find out the cause of the hemorrhage ... We can do more if we have the education you know. (P009)

The nurse and midwife supervisors indicated that they participated in some training at least once a month in case management discussions with their colleagues, or by receiving updates from the reproductive health coordinators. The education activities occurred during the facility Progress Report Submission. Each health facility is required to submit data on selected quality indicators such as the numbers of infants immunized, family planning, births, and maternal and infant mortalities, among others.

When we submit our monthly facility progress reports at the County office, we exchange ideas. For example, if someone had difficulty in dealing with a problem, we discuss amongst each other how to solve it. At these meetings, we also have CME to update on any new or revised policies and practices. (P015)

Some nurses and midwives, especially those in supervisory or management positions, received training during the new health policy or policy revision. This training occurred in a central area. The expectation was that the nurses and midwives who attended the training would disseminate the new knowledge to the other nurses and midwives in their facilities.

We normally have CME when there is a new policy being rolled out. At least one nurse from each health center attends and they must give feedback to the rest of us when they return. We normally hold a meeting where they provide us with this feedback. Then they also provide us with training about the new policy (P005).

Inversely, while some participants stated that they received equitable opportunities for training, others expressed concerns with the irregularly distributed training opportunities amongst the nurses and midwives, and the lack of transparency in selecting staff who attended the training. Due to the per diem payment for attendance of some training sessions, it was likely that other healthcare 
professionals who did not require the training had priority to attend the activity regardless of its relevance to their job. Participants indicated that staff that legitimately needed the knowledge missed the opportunity to be trained, and as well, there was potential misuse of the already scarce resources.

From my experience, I don't find it to be an equal opportunity as such [training opportunities]. I think the seniors take the opportunities and then if there are any other opportunities left, the new employees now may be considered ... The problem around here, these training [sessions] come with money. Yeah, that's what it is. So, if you go there, it's not about knowledge. OK, knowledge is there, you will get the knowledge definitely (laughs) but at the end of the training, you get some per diem and some small money which adds up actually and becomes big money let's say at the end of two weeks, or sometimes six weeks. Yeah. (P012)

Nurses and midwives require regular current evidence-based information to provide adequate and relevant education to their clients. For example, the teaching on family planning, identifying and responding to signs and symptoms of obstetric and neonatal complications.

Immediately after delivery, we educate them on personal hygiene, if there is a tear or episiotomy, how to take care of it. How to watch for danger signs. We educate them on how to breastfeed the baby, how to latch, how many times to breastfeed the infant, how to do exclusive breastfeeding not including water unless it's only in medicine. We also give them information on family planning. We give them immunization information for the baby, and also what danger signs to watch for on herself and the baby and when to return to the hospital. (P012)

The lack of essential supplies, especially in the health centres, caused underutilization of their nurses and midwives' skills. As the following participant explains,

We can insert here [the Intrauterine Device for family planning] - like all of us have gone for the training, but we don't have insertion sets. This sometimes creates those missed opportunities because some mothers can't travel to the referral hospital and so they end up being pregnant again sooner than they wanted to. $(P 011)$

The study found that there was no standardized procedure on how to offer continuing professional development to healthcare professionals in the County. Additionally, the power imbalance created by the lack of a transparent process in the distribution of on-the-job training opportunities disempowered some nurses and midwives' environment. Nurses and midwives require empowerment to provide an empowered environment for their clients.

\section{Discussion}

Continuing professional development (CPD) is a lifelong learning process necessary for all nurses and midwives to keep up with the ever-changing health en- 
vironment by developing personal and professional skills and qualities needed throughout their careers. Being the largest group of healthcare providers, nurses, and midwives has a critical role in the overall quality of patient care, hence maintaining competence in their practice. A well-prepared nursing and midwifery workforce could have the capacity to transform their clients' health outcomes through the application of evidence-based practices [22].

All health professionals in Kenya are required to complete CPD [23]. Nurses and midwives in Kenya engage in both formal and non-formal learning. Although there were several modes of CPD sessions such as organized classroom events in the form of workshops or seminars, held either in the workplace or offsite, there were no transparent processes of ensuring equitable access to all nurses and midwives' learning opportunities. For example, shortages of staff inhibited nurses and midwives from participating in the training sessions. Some indicated that they were not able to attend the training because of family commitments.

Findings from this study indicated no standard training guidelines for nurses and midwives and a lack of clear structure in implementing new policies, processes, and procedures, which required standardized implementation of healthcare protocols and interventions. This lack of standardized healthcare protocols makes it challenging to evaluate the effects of learning and the gap between knowledge and practice for the nurses and midwives.

Similarly, in their study, [24] concluded that midwives in Kenya valued CPD. Still, lack of a formalized structure for topic selection led to a limited or repetitive selection of topics, causing midwives to take courses that are not consistent with their practice area. They continued to say that midwives were not involved in the planning for their CPD activities, causing some not to participate due to time constraints and flexibility issues around family commitments [24]. The course completion demand to meet the professional's bodies' requirements infringes on nurses' and midwives' work and personal life outside of work, creating a sense of disempowerment. Hence, CPD platforms should allow nurses and midwives to choose the time, pace and place of the delivery of learning [25].

CPD courses are now available online for the nurses and midwives in Kenya to access through mobile applications. For example, Kenya's nursing council promoted free CPD courses in 2020 offered by the world continuing education alliance for nurses/midwives [26]. These online courses could not be timelier during the coronavirus disease (COVID-19) pandemic. However, besides internet access limitation, these courses are only available through some mobile devices limits to access to only those who have these particular mobile devices. Further, it is not clear if these courses are context-specific. The learning activities that individuals engage in the workplace are context-specific influences that could benefit both individuals and organizational goals [25].

Other applications such as Safe Delivery are now available to provide support and information to healthcare providers on dealing with obstetric issues [27]. 
Access to such technology would be appropriate in Kenya given that most people have access to a cell phone. The app uses videos, flashcards, and emergency protocols to provide step-by-step instructions on dealing with obstetric issues in a low-resource setting [27]. During the perinatal period, complications during the postpartum period contribute to high mortality and morbidity rates for mothers and their neonates. Hence access to best practices on postpartum management is necessary to curb these mortalities.

Failure to meet the health needs of women and infants in Kenya calls for an intersectionality lens, which critically looks at all intersecting elements to address issues related to the assessment, planning, development, and implementation of the CPD protocols. This study identified intersection elements that created barriers to CPD, such as lack of supervisor support, lack of time, limited access to computers and the internet, and limited financial resources were consistent with those identified by previous studies. Unavailability of dedicated time to learning due to staff shortages and increased workload contributed to the challenges to CPD participation in South Africa [28]. Even in developed countries such as Canada, nurses working in remote areas are hindered by time to attend educational events, since in most cases, they work alone [29]. Similarly, lack of appropriate and accessible professional education and lack of employer and supervisor support were among Australian nurses' barriers [30].

Given the high volumes of maternal and infant deaths in Kenya and other low-middle income countries, it is essential to meet the nurses, and midwives' learning needs working in rural areas. Although most of the nurses and midwives had received training on basic emergency obstetric and newborn care, they were unclear about their responsibilities in implementing healthcare policies, processes, and guidelines, such as the postpartum guidelines [17]. The lack of clarity to policies could cause extreme power imbalances between nurses and midwives and other healthcare providers [31]. Hence, there is the need to ensure CPD guidelines that include implementation structures and the monitoring and evaluation process. This process should also support sharing information with other healthcare professionals and visibility in the decision tables to enable policies that empower and capacity building of the nurses and midwives [32].

\section{Limitations}

This study had some limitations. First, the study examined nurses and midwives working in rural areas of one County in Kenya. Although generalizability is not a guiding principle in qualitative studies, findings from this study may not be generalizable, given the small participant sample. Second, the nurses and midwives participated in a national strike during the data collection period, which could have affected their participation and response to the interviews. Finally, the study did not include nurses who worked in the private sector and other healthcare professionals. Including them could have generated a more stimulated knowledge generation. 


\section{Conclusion}

There is a requirement for more research on the nurses' and midwives' participation in CPD in Kenya, and the effect of CPD on specific health outcomes, including the CPD policy development that ensures context-appropriate courses. That meets the needs of different learners to support the nurses' and midwives' self-reflection in prioritizing their learning needs. The CPD curriculum developers must consider other modes of administering the learning to ensure better uptake and practical knowledge and skills development for the nurses and midwives in rural areas of developing countries, such as Kenya. The intersecting barriers must be considered when developing CPD programs to ensure equitable and accessible learning opportunities for the nurses and midwives, regardless of the location of their primary work area.

\section{Conflicts of Interest}

The authors declare no conflicts of interest regarding the publication of this paper.

\section{References}

[1] World Health Organization. Strengthening the Nursing and Midwifery Agenda Globally.

https://www.who.int/activities/strengthening-the-nursing-and-midwifery-agenda-gl obally

[2] World Health Organization (2012) Enhancing Nursing and Midwifery Capacity to Contribute to the Prevention, Treatment, and Management of Non-Communicable Diseases. Human Resource for Health Observer.

https://www-who-int.proxy.bib.uottawa.ca/hrh/resources/observer12.pdf?ua=1

[3] World Health Organization (2017) Optimizing the Contributions of the Nursing and Midwifery Workforce to Achieve Universal Health Coverage and the Sustainable Development Goals through Education, Research, and Practice. Human Resources for Health Observer. https://www.who.int/hrh/resources/health-observer22/en

[4] Tyer-Viola, L.A., Timmreck, E. and Bhavani, G. (2013) Implementation of a Continuing Education Model for Nurses in Bangladesh. Journal of Continuing Education in Nursing, 44, 470-476. https://doi.org/10.3928/00220124-20130816-07

[5] Manley, K., Martin, A., Jackson, C. and Wright, T. (2018) A Realist Synthesis of Effective Continuing Professional Development (CPD): A Case Study of Healthcare Practitioners' CPD. Nurse Education Today, 69, 134-141. https://doi.org/10.1016/j.nedt.2018.07.010

[6] The International Council of Nurses. International Council of Nurses Code of Ethics for Nurses.

https://www.icn.ch/sites/default/files/inline-files/2012_ICN_Codeofethicsfornurses \%20eng.pdf

[7] Nursing Council of Kenya. Continuing Professional Development Framework (CPD) for Nurses in Kenya. https://www.hrhresourcecenter.org/node/5056.html

[8] Continuing Professional Development.

https://www.nursingmidwiferyboard.gov.au/registration-standards/continuing-prof 
essional-development.aspx

[9] FAQs: Quality Assurance.

https://www.cno.org/en/myqa/faqs-quality-assurance

[10] Guba, E.G. and Lincoln, Y.S. (1994) Competing Paradigms in Qualitative Research. In: Handbook of Qualitative Research, SAGE, Thousand Oaks, 105-117.

[11] Foucault, M. (1980) Power/Knowledge: Selected Interviews and Other Writings 1972-1977. Harvester Press, London.

[12] Lincoln, Y.S. and Guba, E.G. (2000) Paradigmatic Controversies, Contradictions, and Emerging Confluences. In: Handbook of Qualitative Research, 2nd Edition, SAGE, Thousand Oaks, 333-355.

[13] Manojlovich, M. (2017) Power and Empowerment in Nursing: Looking Backward to Inform the Future. Online Journal of Issues in Nursing, 12, 2.

[14] Roper, J.M. and Shapira, J. (2000) Ethnography in Nursing Research. SAGE, Thousand Oaks. https://doi.org/10.4135/9781483328294

[15] Higginbottom, G.M.A., Pillay, J.J. and Boadu, N.Y. (2013) Guidance on Performing Focused Ethnographies with an Emphasis on Healthcare Research. The Qualitative Report, 18, 1-16. https://doi.org/10.7939/R35M6287P

[16] Patton, M.Q. (2002) Qualitative Research and Evaluation Methods. 3rd Edition, SAGE, Thousand Oaks.

[17] Kemei, J.J. (2019) Experiences of Nurses and Midwives Regarding Postpartum Care in Rural Kenyan Communities: A Qualitative Focused Ethnography Study. PhD Thesis, University of Ottawa, Ottawa.

[18] Braun, V. and Clarke, V. (2006) Using Thematic Analysis in Psychology. Qualitative Research in Psychology, 3, 77-101. https://doi.org/10.1191/1478088706qp063oa

[19] Lincoln, Y.S. and Guba, E.G. (1985) Establishing Worthiness. In: Naturalistic Inquiry, SAGE Publications Inc., Beverly Hills, 289-331.

[20] Stronach, I., Garratt, D., Pearce, C. and Piper, H. (2007) Reflexivity, the Picturing of Selves, the Forging of Method. Qualitative Inquiry, 13, 179-203.

https://doi.org/10.1177/1077800406295476

[21] Bierema, L. (2015) Critical Human Development to Enhance Reflexivity, Change Discourse, and Adopt a Call-to-Action. Human Resource Development Review, 14, 119-124. https://doi.org/10.1177/1534484315585206

[22] Gerrish, K., Guillaume, L., Kirshbaum, M., McDonnell, A., Tod, A. and Nolan, M. (2011) Factors Influencing the Contribution of Advanced Practice Nurses in Promoting Evidence-Based Practice among Front-Line Nurses: Findings from a Cross-Sectional Survey. Journal of Advanced Nursing, 67, 1079-1090. https://doi.org/10.1111/j.1365-2648.2010.05560.x

[23] Ministry of Health. National Continuing Development Regulatory Framework. https://uasingishureproductivehealth.files.wordpress.com/2015/08/continuing-profe ssional-development-framework-2014.pdf

[24] Gitonga, L. and Muriuki, S.N. (2014) Perspectives of Continuing Professional Development (CPD) for Kenyan Midwives. Open Journal of Clinical Diagnostics, 4, 89-100. https://doi.org/10.4236/ojcd.2014.42015

[25] Alsop, A. (2013) Continuing Professional Development in Health and Social Care: Strategies for Lifelong Learning. Wiley-Blackwell, Hoboken.

https://doi.org/10.1002/9781118782859

[26] The World Continuing Education Alliance. New Online Free CPD Platform. https://www.icn.ch/what-we-do/projects/world-continuing-education-alliance-wcea 
[27] (2019) Safe Delivery. Obstetrician \& Gynaecologist, 21, 152. https://doi.org/10.1111/tog.12564

[28] De Villiers, M. (2008) Global Challenges in Continuing Professional Development: The South African Perspective. Journal of Continuing Education in the Health Professions, 28, 25-26. https://doi.org/10.1002/chp.205

[29] Penz, K., D’Arcy, C. and Stewart, N. (2007) Barriers to Participation in Continuing Education Activities among Rural and Remote Nurses. Journal of Continuing Education in Nursing, 38, 58-66. https://doi.org/10.3928/00220124-20070301-03

[30] Ross, K., Barr, J. and Stevens, J. (2013) Mandatory Continuing Professional Development Requirements: What Does this Mean for Australian Nurses. BMC Nursing, 12, Article No. 9. https://doi.org/10.1186/1472-6955-12-9

[31] Kemei, J. and Etowa, J. (2018) Understanding the Sociocultural Context of Postpartum Nursing Care in Rural Kenya Using a Critical Theory Lens. The Humanities and Social Sciences Review (HSSR), 8, 61-74.

[32] Etowa, J. (2014) Painting the Landscape: Is the Invisibility of Nigerian Nurses in Research and Policy Development Arenas Ailing Healthcare in the Country? Journal of Pregnancy and Infant Health, 1, e105.

https://doi.org/10.4172/2376-127X.1000e105 